\title{
Beta to Omega Phase Transformation in a Ti-Mo Alloy Deformed in an Impact Compression Mode Due to Aging
}

\author{
E. Sukedai*, D. Yoshimitsu*, M. Kiritani** and H. Hashimoto* \\ * Okayama University of Science, Okayama 700-0005, JAPAN \\ ** Hiroshima Institute of Technology, Hiroshima 713-5193, JAPAN
}

Nucleation-sites of phase transformations in many kinds of materials have been considered as lattice defects such as dislocation lines, vacancy clusters, and atomic steps on surfaces, inclusions and so on. In many kinds of beta Ti alloys, beta to omega phase transformations occur due to quenching, stressing, heating and cooling. However, nucleation-sites of the phase transformations are not necessary clear. On the other hand, it has been reported that when metallic materials are deformed by an ultra high speed loading, the plastic strain is resulted from motions of vacancies without dislocation motions. It is also considered that lots of vacancies remain in the materials deformed with a high strain rate. Therefore, it is useful for considering nucleation-sites of phase transformations that phenomena of beta to omega phase transformation in rapidly deformed materials due to aging are investigated, and roles of vacancies to nucleation-sites of the phase transformation will be discussed.

In the present work, a beta -Ti alloy deformed in an impact compression mode was aged, and the appearance behaviours of omega phase crystals and their morphology were investigated using electron microscope techniques.

A Ti-20mass\%Mo alloy plate was solution-treated at $1223 \mathrm{~K}$ for $4.5 \mathrm{ks}$. Disks with $3 \mathrm{~mm}$ in diameter were prepared by a spark erosion machine. Several disks were deformed in an impact compression mode with a strain rate, approximately $104 / \mathrm{sec}$. Also, some disks were deformed statically. True strains in both cases were approximately $100 \%$. Disk specimens deformed in both modes were aged at $623 \mathrm{~K}$ for $28.8 \mathrm{ks}$. Thin foil specimens of as-deformed disks and aged disks were prepared by an electro-polishing method. A JEM 4000EX electron microscope was used.

Figure 1 shows a weak beam dark field image of an as-deformed specimen in an impact compression mode. Straight and isolated dislocation lines can be seen, but dislocation tangle are hardly observed. These features consist with a characteristic of dislocation configurations observed in materials deformed with a high strain rate. And lots of white dotes can be seen. This result suggests there remain vacancies and vacancies clusters. Figure 2 shows a dark field image of omega phase crystals in a specimen deformed in an impact compression mode, followed by aging at $623 \mathrm{~K}$ for $28.8 \mathrm{ks}$. Although it is well known that aged omega phase crystals in this alloy have a spheroid shape, the shape of many omega phase crystals shown in Figure 2 is not a spheroid. Their shape are string like. This kind of shape was not observed in a specimen deformed in a static mode, followed by the same aging. Figure 3 shows a high magnification dark field image of a string-like omega phase crystal. In order to confirm its atomic structure, a high resolution image of this string-like omega phase crystal was taken. Figure 4 shows a processed image from the image, and an atomic structure of omega phase crystal is recognized. These results mentioned above suggest that vacancies remained in a rapidly deformed specimen affect the formation of omega phase crystals. 


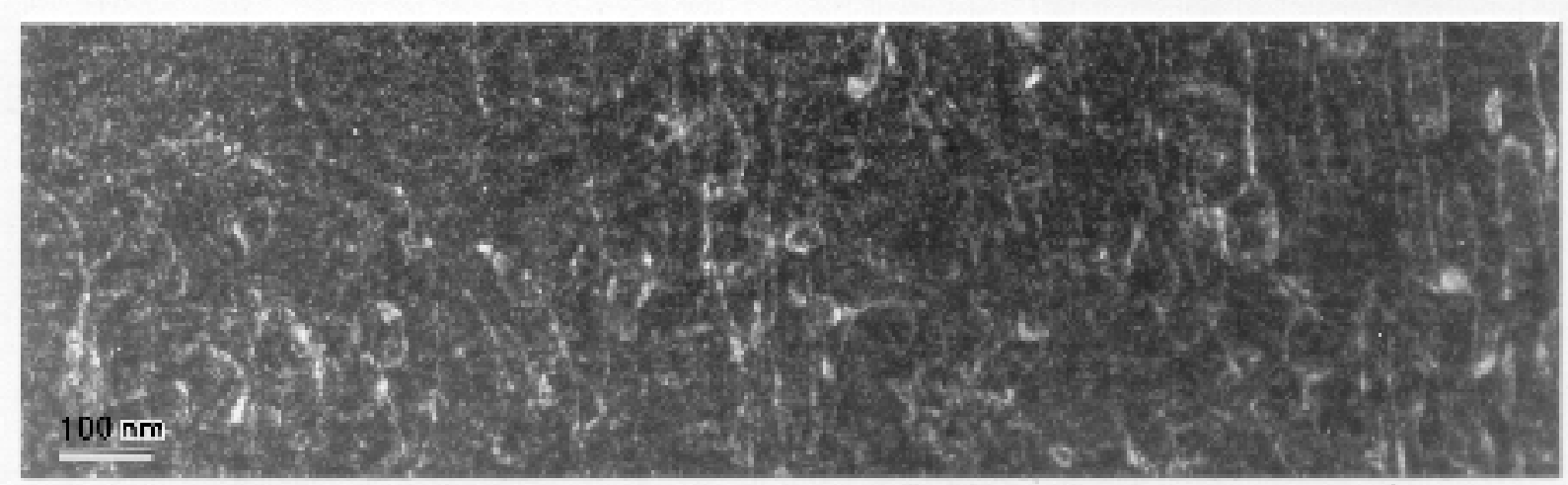

FIG. 1 A weak beam dark field image of an as-defonned Ti-20Mo alloy in an impact mode.

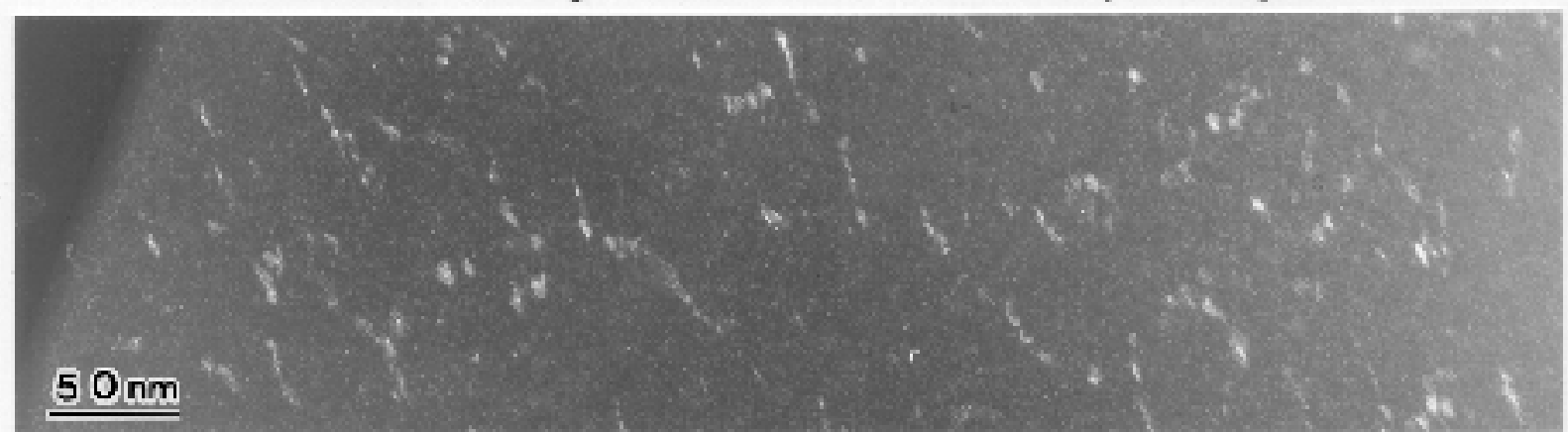

FIG. 2 A dark field image of omega phase erystals in a Ti-20Mo alloy deformed in an impact compression mode, followed by aging at $623 \mathrm{~K}$ for $28.8 \mathrm{ks}$.

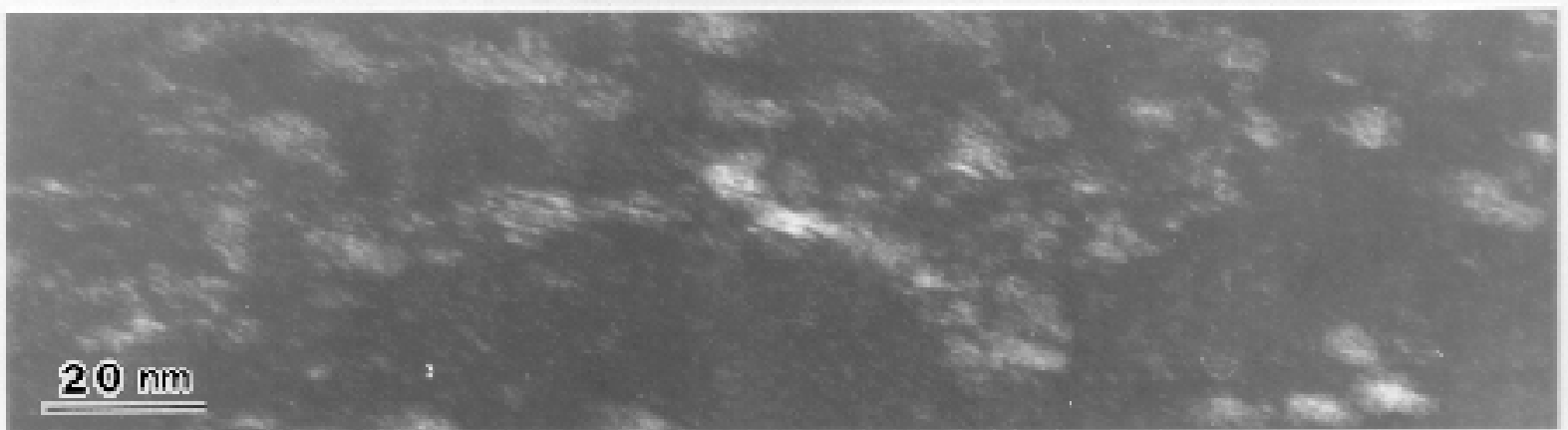

FIG. $3 \mathrm{~A}$ high magnification dark field image of a string-like ome gea phase crystal.

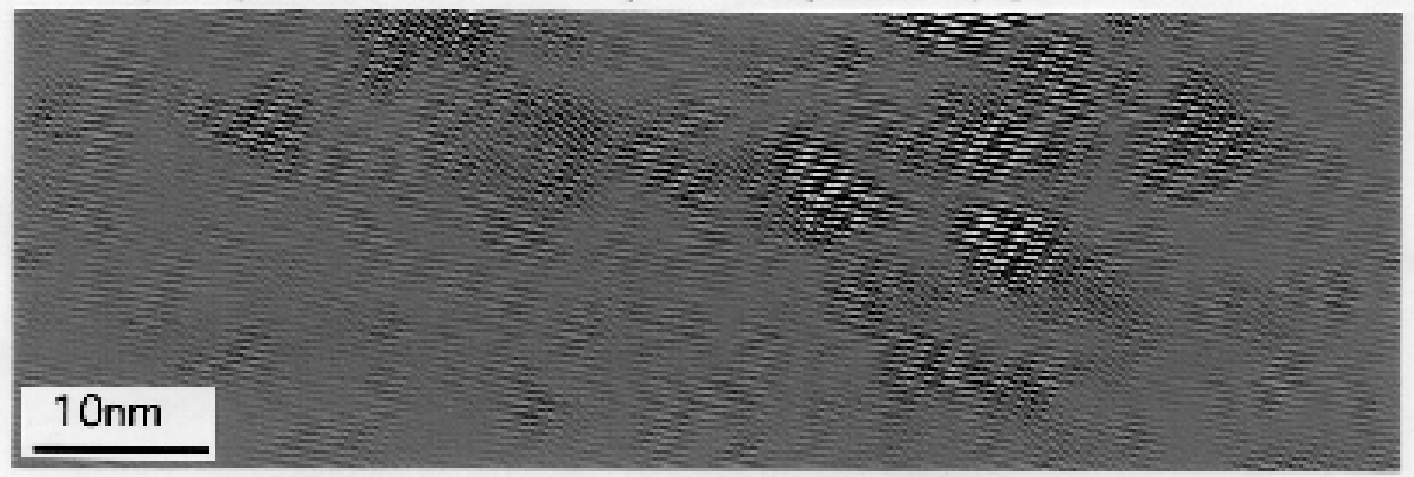

FIG. 4 A processed HREM image of the string-like omega phase crystal shown in Fig. 3, which is showing an atomic structure of omega planse crystal. 